\title{
Top Legal Risks and Regulatory Trends Facing Canadian Optometrists
}

\section{GOWLING WLG bmS.}

This article was prepared and written by the BMS Group Healthcare Professionals Insurance Alliance legal team at Gowling WLG (Canada) LLP (Gowlings), one of the largest and most highly-recognized legal firms in medical defence and professional liability in Canada. This article is meant to be a general overview of the claims and trends that are applicable to the optometry profession. Should you have any specific concerns or questions about an incident or aspect of your practice, please contact the CAO, your regulator or the pro bono legal advice hotline. This article is not legal advice and should not be considered as such. In the event of an actual or potential professional liability claim, CAO members who participate in the Professional Liability Insurance program are eligible for 30-minute pro bono and inclusive legal claims defence services from Gowlings.

$\mathrm{D}$ octors of Optometry are subject to many of the same legal risks and trends facing most regulated health professionals in Canada. These legal exposures range in scale from College investigations into an optometrist's billing practices to seven-figure lawsuits alleging that an optometrist failed to appropriately diagnose and refer a patient for further investigation and treatment. This article uses information gained through calls made by insured optometrists to the Canadian Association of Optometrist (CAO)'s Insurance Program pro bono legal services hotline along with over 15 years of program claims data to provide an overview of common legal risks and regulatory trends affecting the profession.

The good news is that there is a low chance that you as an optometrist will require legal assistance regarding your professional practice. However, in the event that you do require such legal assistance, it is most likely because you have been named in a complaint made to your provincial regulatory body rather than because of any kind of civil or criminal action. Complaints to regulators comprise the majority of matters that are handled by legal counsel for optometrists participating in the CAO Insurance Program, and this is consistent with what we see across many other regulated health professions in Canada.

COLLEGE COMPLAINTS:

While navigating a complaint and/or investigation from your regulator may seem fairly straightforward, you need to be aware of the significant powers that provincial regulators have that can impact your ability to practice. For this reason, you should always communicate with your insurance broker or insurer before responding to an inquiry from your regulator. Your insurance representative will examine your policy, identify your coverage and assist you with the next steps, including coordinating your legal defence if required.

Optometry regulators often respond to complaints involving billing practices. These include misunderstandings regarding the appropriate amount to be charged, billing for services not covered by provincial health plans, and poor record-keeping practices that lead to the conclusion that a member is billing for services that were not provided. Inaccurate or false billing creates a negative perception of the profession and, as a result, regardless of whether the inaccuracy is intentional or not, ethical concerns abound, and the regulatory penalties can be severe. As a result, inaccurate, false, or misleading billing can lead to disciplinary sanctions, civil liability, and even criminal liability for fraud. 
In a 2018 decision from the College of Optometrists of Ontario, an optometrist was found to have billed for services not provided to two patients. Following an investigation and Disciplinary Hearing, the College imposed a 14-week suspension of the member's license to practice and ordered the optometrist to pay the College's costs in the amount of $\$ 5,000$. As a condition of registration, the member was also required to complete and pass a course on Ethics and Boundaries.

In British Columbia, an optometrist who billed the Medical Services Plan (MSP) for patients who did not have any billable conditions and for services that were billed and paid privately by the patients was recently suspended for 18 months. The College allowed the final nine months of the suspension to be stayed, provided the member successfully completed courses on professional ethics and record-keeping prior to returning to practice. Further, the College mandated that the optometrist return to practice under direct supervision for a period of one year, and that the member cooperate with six random site-based audits by a College-appointed inspector. As provincial regulators continue to focus on this issue, we believe penalties imposed by discipline panels will continue to be significant.

Generally, the conduct that can give rise to these disciplinary measures is the result of a misapprehension or limited consideration of the relevant and applicable standards rather than intentional fraud. Accordingly, the simplest way to avoid billing-related claims is to:

- review all of the relevant guidelines, standards and regulations;

- undertake frequent reviews of billing systems and software with staff; and

- participate in continuing education that focuses on the interpretation and understanding of guidelines.

It is also important to remember that billing is a reflection of the entire scope of service that you, as an optometrist, provide. To invoice in accordance with your professional standards, you must also understand and apply all of the guidelines including those not directly related to billing, such as record-keeping, informed consent and delegation.

EMERGING RISKS:

Two additional emerging risks facing optometrists include the use of social media and sexual abuse/unprofessional behavior.

SOCIAL MEDIA

The number of regulated professionals who use social media continues to increase, particularly in health care. While social media offers an unprecedented marketing platform for regulated professionals and serves as a convenient and cost-effective way to share ideas and industry trends with other professionals and clients/patients, its use is not without risk. Regulatory bodies are increasingly challenged by registrants using social media in ways that may reflect poorly on the profession, and health care professionals are being disciplined for a variety of issues, including: (i) disclosing confidential information; (ii) making disparaging remarks and allegations, often regarding members of the same profession; and (iii) posting content that contradicts a regulatory body's policies or is unprofessional. We expect that provincial optometry regulators will take the same approach as other regulators across Canada.

Best practice for professionals using social media is to be professional, courteous and respectful in all online activities. The high standard of behavior expected of a professional on social media also extends to your use of personal media. 


\section{SEXUAL ABUSE/UNPROFESSIONAL BEHAVIOR}

We anticipate that there will be a rise in complaints related to sexual abuse and/or unprofessional behavior in the coming years, as provincial regulators become more vigilant with investigations into these activities. This is due in part to a perception that provincial regulators were not taking cases of sexual abuse/inappropriate behavior as seriously as they should have and in part as a response to the current climate. As patients become more comfortable raising concerns about how they have been (or feel they have been) treated by their health care professionals, we anticipate that number of these types of complaints will continue to rise.

As an example of how serious the consequences can be for making inappropriate comments to a patient, in the past year, the College of Optometrists of Ontario suspended a member for five weeks (and imposed other penalties on the member) for making inappropriate sexual comments to a patient during the course of an appointment. The Ontario regulator is also in the process of determining penalties to be imposed on an optometrist who was recently found guilty of sexually abusing three patients who were also his employees. The member faces the possibility of having his license suspended for four months. As well as completing an ethics course, he may also be required to pay $\$ 30,000$ to the College for investigation and legal costs and \$43,000 towards the therapy and counselling for the employees he abused.

As a regulated health professional, you must ensure that your interactions with patients remain professional and courteous at all times, whether face-to-face or online. Comments that you may have considered harmless in the past are no longer acceptable. Patients are more able and willing to voice their concerns and hold you to account if they feel you have acted inappropriately.

\section{Helpful contacts for optometrists participating in the CAO member insurance program:}

Report a professional liability insurance claim or College complaint - Contact BMS Canada Risk Services Ltd. (BMS Group) at 1-844-517-1371 or cao.insurance@bmsgroup.com

Pro bono legal advice program - Contact Gowling WLG (Canada) LLP at 1-844-792-2022 